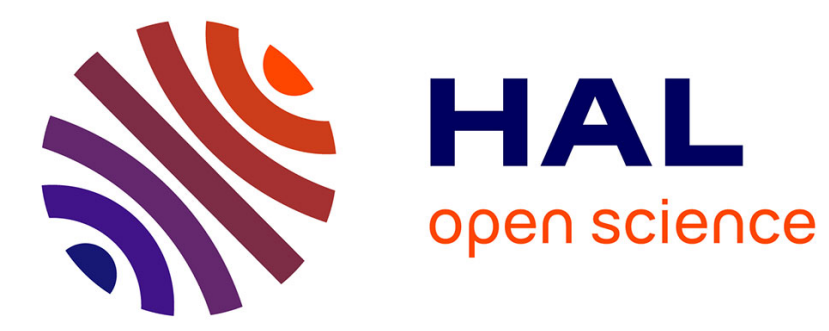

\title{
Mobilisations des migrants en Europe: du national au transnational
}

Riva Kastoryano

\section{To cite this version:}

Riva Kastoryano. Mobilisations des migrants en Europe: du national au transnational. Revue Europeenne des Migrations Internationales, 1994, 10 (1), pp.169-181. hal-01016777

HAL Id: hal-01016777

https://hal-sciencespo.archives-ouvertes.fr/hal-01016777

Submitted on 1 Jul 2014

HAL is a multi-disciplinary open access archive for the deposit and dissemination of scientific research documents, whether they are published or not. The documents may come from teaching and research institutions in France or abroad, or from public or private research centers.
L'archive ouverte pluridisciplinaire HAL, est destinée au dépôt et à la diffusion de documents scientifiques de niveau recherche, publiés ou non, émanant des établissements d'enseignement et de recherche français ou étrangers, des laboratoires publics ou privés. 


\section{Mobilisations des migrants en Europe : du national au transnational}

In: Revue européenne de migrations internationales. Vol. 10 N¹. Mobilisations des migrants en Europe - Du national au transversal. pp. 169-181.

Citer ce document / Cite this document :

Kastoryano Riva. Mobilisations des migrants en Europe : du national au transnational. In: Revue européenne de migrations internationales. Vol. 10 №1. Mobilisations des migrants en Europe - Du national au transversal. pp. 169-181.

doi : 10.3406/remi.1994.1397

http://www.persee.fr/web/revues/home/prescript/article/remi_0765-0752_1994_num_10_1_1397 


\section{Résumé}

Mobilisations des migrants en Europe : du national au transnational

\section{Riva KASTORYANO}

II est devenu habituel d'opposer des « modèles » nationaux lorsqu'il s'agit de comparer les formes d'organisation et d'intégration des migrants des années 1960 installés dans différents pays européens. Ces modèles fondés sur les traditions politiques et les relations que chacun des états entretient avec les populations en situation de minorité (religieuse, ethnique, raciale) influencent la mobilisation des populations en question. En effet, leurs modes de mobilisation dénotent dans chacun des pays l'intériorisation des normes et des conduites politiques propres à chacun des pays et soulignent dès lors la « nationalisation » de leurs actions. Or, la construction de l'Europe remet en cause la validité et la pertinence des modèles. Le mode de participation politique des populations issues de l'immigration trouve un appui dans ce nouvel espace politique qui favorise les solidarités transnationales en fonction de la nationalité (actuelle ou d'origine), la religion, ou la « couleur ». Les actions qui les accompagnent contournent les politiques nationales pour aboutir à une représentativité au niveau européen.

\section{Abstract \\ Transnational Solidarities of Migrants in Europe \\ Riva KASTORYANO}

A comparative study on the settlement of immigrants in different European countries emphasizes the " models " that each country develops as the way to deal with a minority group. These models based on each country's political traditions and its conception of nationhood and citizenship obviously has an effect upon immigrants, leading to their mobilization through the creation of voluntary associations. The result might be called the " nationalisation » of their political action. Projects for European Unity however, challenge the validity of these national models. In fact, migrants or minorities (the terminology varies from one country to another) are developping new networks based on their nationalily (actual or of origin), their religion or race in order to promote their political participation beyond national boundaries. Their aim is the search for representativity on a European level.

\section{Zusammenfassung \\ Transnationale Solidaritäten der Migranten in Europa \\ Riva KASTORYANO}

Es ist üblich geworden, von « nationalen Modellen » zu sprechen, wenn es um den Vergleich der Organisations - und Integrationsformen von Migranten geht, die sich seit den 60er Jahren in verschiedenen europäischen Ländern niedergelassen haben. Diese Modelle, die auf politischen Traditionen und dem Verhältnis beruhen, das der Staat zu Bevölkerungsgruppen in der Position von Minderheiten (seien sie es aufgrund der Religion, der Ethnie oder der Rasse) unlerhält, beeinflussen die Mobilisierung dieser Gruppen. Ihre Mobilisierungsformen weisen in jedem Land auf die Interiorisierung der jeweiligen politischen Normen und Verhaltensregeln hin und sind so ein Zeichen der " Nationalisierung » ihrer Aktionen. Die europäische Vereinigung stellt aber den Einfluss und die Prägnanz dieser Modelle in Frage. Die politischen Partizipationsformen der Migranten passen sich dem neuen politischen Feld an, das transnationale Solidaritäten bevorteilt, die sich aufgrund der Nationalität (der jetzigen oder der Herkunftsnationalität), der Religion oder der « Farbe » bilden. Die Aktivitäten, die damit einhergehen, umgehen die nationale Politik, um eine europäische Representativität zu erlangen.

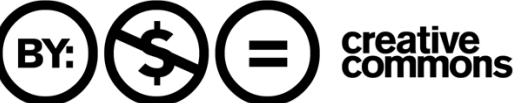


Revue Européenne des Migrations Internationales Volume 10 - No 1 1994

\title{
Mobilisations des migrants en Europe : du national au transnational
}

\author{
Riva KASTORYANO
}

L'objectif déclaré de l'Europe est, d'après l'Acte Unique de 1985 , celui « d'un espace sans frontières intérieures dans lequel la libre circulation des marchandises, des personnes, des services et des capitaux est assurée ". L'Europe ainsi définie est a priori associée à l'idée d'ouverture qu'exige la création d'un grand marché économique. Le traité de Maastricht signé en septembre 1992, en introduisant une dimension politique à la construction de l'Europe, jette une ombre sur cette idée d'ouverture élaborée par l'Acte Unique. Depuis lors, l'Europe renvoie à l'image d'une forteresse pour exprimer sa fermeture vis-à-vis de l'extérieur. Ses frontières se dessinent non seulement à travers les limites géographiques, cependant variables, mais aussi à travers la recherche d'une unité politique. Cela conduit à des interrogations sur l'identité européenne et à une redéfinition du concept de citoyenneté projeté dans le nouvel espace politique en construction qui entraînent des inclusions et des exclusions de fait.

La citoyenneté européenne telle qu'elle est définie par le traité de Maastricht projette au niveau européen sa conception nationale. Le droit de vote local dans leur lieu de résidence, attribué aux individus ayant la nationalité d'un des pays de l'Union Européenne, stipulé par le traité, accentue le fossé entre autochtones (immigrés européens et nationaux) et immigrés étrangers (non-européens) : les Maghrébins, les Turcs, les Africains, les Pakistanais, les Indiens, les Surinamiens, en France, en Allemagne, aux Pays-Bas, en Grande-Bretagne. On évalue à 13 millions le nombre "d étrangers" (non européens) vivant en situation régulière dans les douze pays de la Communauté Européenne(1). Cette population, aussi diversifiée culturellement, sinon plus, que l'Europe Unie elle-même, installée dans pratiquement tous les pays européens depuis les années 1960, se mobilise pour faire reconnaître ses particularismes identitaires aussi bien au niveau national que communautaire. 
Au niveau national, les mobilisations autour de l'identité donnent lieu à des négociations entre les associations créées dans ce but et les pouvoirs publics. Les interactions permanentes entre les groupes et l'appareil d'Etat conduisent à des constructions des " modèles nationaux " qui cherchent à mettre en évidence des spécificités puisées dans les principes fondateurs des États-nations. L'hétérogénéité de ces modèles est bien mise en valeur par la variété des termes qui désignent ces populations, termes qui se réfèrent aux représentations idéologiques des Étatsnations, aux politiques d'immigration et d'intégration : " population immigrée ou issue de l'immigration " en France ; "étrangers " en Allemagne, "minorités " aux Pays-Bas, " communautés ethniques " en Grande-Bretagne.

Cependant, même si les politiques d'immigration et d'intégration relèvent des compétences nationales, le mode de participation politique des populations immigrées trouve un point d'appui dans le nouvel espace politique européen. Les populations issues de l'immigration élaborent de plus en plus des solidarités " primordiales " qui se dessinent à travers les frontières nationales en fonction de la nationalité (actuelle ou d'origine), de la religion ou encore de la " race ". Les actions qui les accompagnent contournent les politiques nationales pour aboutir à une représentativité au niveau européen. Certains parlent déjà de la " $13^{\mathrm{e}}$ population " ou du " $13^{\mathrm{e}}$ État " ou même de " $13^{\mathrm{e}}$ nation " pour souligner l'importance de l'organisation communautaire transnationale des résidents européens différenciés de fait des " citoyens européens".

Cette évolution oriente la réflexion sur la pertinence des modèles nationaux quant à la mobilisation des populations issues de l'immigration et à leur mode d'intégration dans les sociétés européennes. La recherche d'une représentativité au niveau européen et les revendications mises en avant mettent en évidence le dépassement des cadres nationaux. La question est de savoir si l'Union Européenne constitue, pour chacun des États-nations, une pression poussant à redéfinir ses relations avec les populations " immigrées " d'une part, et si les nouvelles formes de solidarité élaborées par ces dernières aboutissent dans les faits à la constitution de " minorités " en Europe.

\section{ORGANISATION DES IDENTITÉS ET MOBILISATIONS}

Les associations apparaissent comme les nouvelles formes d'organisation qui conduisent les groupes latents à l'action. Les groupes se forment à partir d'une nationalité commune, ou d'une religion commune. Tout en maintenant leur système culturel dans leur mode d'organisation, ils adoptent des stratégies d'action en relation avec les institutions de la société d'installation. Leur but proclamé est de définir une identité de groupe séparé. Mais en réalité, les associations répondent à un double objectif : l'affirmation d'une identité collective et son intégration dans la société globale et ses institutions. D’après $J$. Rex, une nouvelle identité, quil appelle " une identité de citoyenneté " $\left({ }^{2}\right)$, se crée et s'élabore à partir de cette double stratégie, où la communauté définit ses frontières d'identité et d'action en liaison avec les institutions nationales. 


\section{AFFIRMATION D'UNE IDENTITÉ}

Au niveau local, les associations s'appuient sur les solidarités religieuses, nationales et/ou ethniques. Elles contribuent à créer des " communautés " à part, à institutionnaliser leur identité, à en négocier chacun de ses éléments constitutifs avec l'appareil d'État (3). Les associations permettent donc aux populations immigrées d'affirmer une identité collective et d'agir en tant que groupe de pression au niveau national. Leur action porte essentiellement sur la lutte contre les discriminations raciales et ethniques dans les domaines du logement, de l'enseignement, de l'emploi. Pour mieux défendre les droits des immigrés, elles ont comme objectif d'obtenir une véritable reconnaissance, une représentativité politique.

En Grande-Bretagne, la recherche d'une représentativité communautaire suscite de nouvelles identifications des populations qui se définissent comme " ethniques ". Par exemple, l'Association des Travailleurs Indiens, (IWA Indian Workers Association), et l'Organisation de la Jeunesse Progressiste (Progressiste Youth Organisation), a priori une association locale située à l'est de Londres, sont significatives par l'ampleur de leur mobilisation à base " ethnique ". Bien que les adhérents de l'organisation des jeunes progressistes soient des Bangladeshi de Tower Hamlet à Londres, ou que ceux de l'association des travailleurs indiens soient surtout des Penjabi, leurs actions se conjuguent et les deux communautés se reconnaissent dans l'identité Black. Telle devient l'expression de la lutte contre le racisme.

En France, que les associations soient organisées au niveau local ou national, ou qu'elles se constituent en fédération, il est difficile de parler a priori d'une mobilisation autour des thèmes qui touchent à l'identité "ethnique " $\left({ }^{4}\right)$, pour reprendre l'expression britannique. Dans l'ensemble, la préoccupation majeure de ces institutions se situe dans le domaine social et le discours de leurs dirigeants porte sur l'élaboration d'une solidarité sociale de quartier associée avec une solidarité nationale et religieuse de ses habitants. Religieux musulmans ou laïques, ils conjuguent leurs actions au niveau local ; ils reprennent le discours centralisateur qui renvoie aux préoccupations de l'État. L'exclusion devient le leitmotiv des discours réciproques. La structure de la ville est cependant un facteur important dans la multiplicité des formes de mobilisation et d'affirmation des identités de groupe. A Marseille, par exemple, la composition " ethnique " de la ville fait qu'on retrouve une multitude d'associations, culturelles, religieuses, des associations de quartiers, des radios " communautaires " qui se définissent toutes plus ou moins comme multiculturelles. Dans ce contexte, Radio-Gazelle, radio locale maghrébine se donne comme mission de " gérer " les conflits entre différentes communautés en essayant d'atténuer les clivages entre elles. Lidéologie laïque qui orientait ses activités a cédé sa place à des préoccupations religieuses à la fois pour répondre à la demande de son public et aussi parce que l'Islam est un élément d'identité commun à toutes les communautés auxquelles la radio s'adresse $\left({ }^{5}\right)$. Ainsi, même si les activités se définissent comme sociales au départ, elles se trouvent "ethnicisées" de fait.

En Allemagne, les associations se regroupent d'après la nationalité de leur membres constituant des fédérations structurant leur action dans la lutte contre les 
discriminations. Les militants revendiquent la reconnaissance de leur présence permanente et l'octroi de la double nationalité comme une garantie de séjour. Ils négocient dans l'ensemble la reconnaissance d'un statut de minorité. Il en est de même au Pays-Bas, où les populations immigrées définies officiellement comme des " minorités " se regroupent pour créer des commissions représentatives par nationalité, à partir des fédérations d'associations. Leur revendication semble cependant loin de porter sur des motivations " ethniques". Leur action est surtout orientée vers les politiques sociales qui favoriseraient l'intégration par le logement, l'emploi, les conditions de scolarisation des enfants des populations qu'elles représentent. L'action des associations, des fédérations d'associations et des commissions consiste dès lors à "lutter contre toute discrimination et toute privation économique ".

Dans chacun des pays, les militants trouvent dans l'identité religieuse, l'élément essentiel de la construction des communautés. Ils élaborent des discours sur l'importance d'une croyance pour une meilleure intégration dans la société(6). Dans certains quartiers des grandes villes françaises, les associations socio-culturelles (terme qui souligne l'idéologie laïque de leurs activités) se trouvent en compétition avec les associations islamiques avec lesquelles elles conjuguent leurs actions afin de rassembler les différents éléments de l'identité collective et dessiner les frontières d'une communauté, plutôt d'une collectivité, ne serait-ce qu'au niveau local comme c'est le cas de Radio Gazelle à Marseille. Cette rivalité entre associations laïques et religieuses renforce les frontières d'une communauté. En Allemagne et aux Pays-Bas, les associations religieuses font partie des fédérations d'associations et des commissions représentatives.

\section{INTÉGRATION NATIONALE}

Ainsi, les associations tout en affichant publiquement des particularismes identitaires développent des stratégies d'intégration dans la société globale et ses institutions. En réalité, ce sont les interactions avec les pouvoirs publics dans chacun des pays qui donnent un contenu aux discours des militants et orientent les actions des associations.

Les États soutiennent les projets des associations lorsqu'ils portent sur l'intégration sociale des migrants. Ils les aident à se mobiliser pour l'enseignement, l'emploi et le logement. Ils financent leur campagne de lutte contre les discriminations raciales et ethniques. Les municipalités prêtent des locaux pour des manifestations culturelles des identités telles que concerts, journées de cuisine ou autres. L'objectif des États est d'avoir un interlocuteur entre les pouvoirs publics et les groupes ainsi constitués.

Les actions collectives entreprises par les migrants sont, par conséquent, souvent en relation avec les institutions locales ou nationales. De par le mode d'organisation et de mobilisation, ils s'adaptent aux règles du jeu établies par les États, utilisent les mêmes outils que les pouvoirs publics dans le processus de négociation de leurs intérêts collectifs. L'objectif des migrants est de désigner un représentant susceptible d'exprimer les intérêts collectifs du groupe pour aboutir à la longue à une représentation politique. C'est ainsi que s'exprime la volonté d'intégration. 
La nécessité de négocier avec l'État conditionne les formes d'organisation et les structures des associations. En Allemagne, pour répondre aux attentes des pouvoirs publics et faciliter ainsi les négociations, des associations turques, par exemple, se constituent en Gemeinschaft, d'après le modèle des Églises. Il en est de même aux Pays-Bas, à la différence que le discours sur le multiculturalisme et une politique définie explicitement comme une politique de "minorité ", en vigueur depuis 1985, ont donné lieu à la formation des commissions représentatives par nationalité. Ces commissions, créées à partir des regroupements de différentes associations, ont un rôle consultatif auprès des autorités publiques néerlandaises. En revanche en France, le Fonds d'Action Sociale, principal pourvoyeur des associations socioculturelles, favorise la création d'associations locales, mais cherche à éviter la formation de communautés par nationalité ou de communautés religieuses $\left({ }^{7}\right)$.

Même si les relations qu'entretiennent les associations avec l'État sont parfois des relations de dépendance, si les parties politiques développent ces relations dans un but électoral, les migrants cherchent, en se mobilisant, à influencer l'opinion publique dans le cadre du débat national sur la reconnaissance de leur identité devenue collective. En Grande-Bretagne, ils ont réussi à sensibiliser les politiques sur une identité Black. Les actions dans ce sens ont pour but de négocier un statut de minorité qui leur permette de maintenir leur propre culture et de se faire entendre dans la vie politique locale et même nationale.

Quant aux associations islamiques, bien qu'autonomes par rapport à l'État, leurs dirigeants développent des stratégies d'action en relation avec les politiques nationales et la place qu'accorde chacun des États européens à la religion majoritaire ou minoritaire $\left.{ }^{8}\right)$. Leur rivalité avec les associations culturelles fondée sur un souci de répondre aux besoins identitaires de la population concernée intègre les associations religieuses aussi dans le débat national. En Allemagne, R. Detalle constate une "germanisation " en cours parmi les immigrés musulmans qui se manifeste non seulement par le caractère local de leurs revendications (enseignement du Coran, construction de mosquées), mais aussi par l'utilisation d'arguments et de stratégies qui témoignent de la volonté de ne plus refuser une action politique $\left({ }^{9}\right)$. Leur mode d'organisation et les négociations qu'ils entreprennent avec les autres associations, mais aussi avec les pouvoirs publics dans chacun des pays d'installation les situent dans le débat général concernant les populations immigrées.

Ainsi les structures d'organisation des associations et les discours de leurs dirigeants reflètent les exigences des États pour assurer l'intégration des populations immigrées. J. Rex appelle " britannisation " ce processus tel qu'il s'exprime dans la mobilisation ethnique des Indiens de l'Association des Travailleurs Indiens ou celle des Penjabi de l'association progressiste des jeunes de Tower Hamlet ( $\left.{ }^{10}\right)$. Il en est de même en France et en Allemagne. De fait la " nationalisation " de la mobilisation est le résultat d'une adaptation aux règles du jeu établies par les États ; les groupes, constitués en associations, qui utilisent les mêmes outils que les pouvoirs publics et les institutions nationales dans la négociation de leurs intérêts collectifs et la reconnaissance de leurs spécificités identitaires. Les militants témoignent ainsi de leur " acculturation politique " pour reprendre le terme d'Haber- 
mas, qui se manifeste par l'adhésion des populations immigrées à la culture politique de leur nouvelle patrie, mais précise l'auteur "sans renier les formes socio-culturelles de leurs origines "( $\left.{ }^{11}\right)$.

Paradoxalement, celà entraîne une convergence dans leur mode d'intégration dans les différents pays européens.

\section{ORGANISATION TRANSNATIONALE DES IDENTITÉS}

Malgré ces caractéristiques "nationales " de la mobilisation, la recherche d'une représentativité s'étend désormais à une échelle européenne, d'autant plus que l'Europe se présente comme un espace ouvert à la revendication des intérêts et des identités. Les populations immigrées s'adressent aux institutions européennes au même titre que les autres organisations qui cherchent à influencer les politiques nationales par leur action à une échelle communautaire. Cela leur permettrait de renforcer leur représentativité à la fois au niveau national et européen. D'où l'élaboration de nouvelles formes de solidarité qui se situent au-delà de la " nationalisation " des actions des " minorités ". Se tissent des réseaux transnationaux et de nouvelles structures de solidarité se dessinent à travers les frontières.

\section{EFFORTS DE COORDINATION}

Les associations ont du mal à coordonner leurs actions et leurs revendications. Même si elles se situent dans la plupart des cas par rapport au débat dans chacun des pays, sur des thèmes comme l'emploi, le logement, le racisme, la xénophobie, ou encore l'exclusion, la citoyenneté, les débats sont devenus européens. La définition d'une action commune transnationale se heurte à de nombreux obstacles. Tout d'abord, la spécificité des situations nationales limite les possibilités d'une mobilisation à l'échelle européenne. La " nationalisation " de leur mobilisation, l'intégration de leur action dans le jeu national, rend difficile la définition des intérêts communs, l'identification commune de la part des acteurs. Par exemple, en Grande-Bretagne, le groupe ethnique est défini par sa couleur, critère objectif, mais aussi subjectif d'identification, en France, la citoyenneté est, en théorie et dans l'idéal, le seul critère de définition du Français et de l'Étranger; l'Allemagne n'étant " pas un pays d'immigration ", en Allemagne être étranger ou même " concitoyen étranger " devient un statut légal dans le discours public, tout comme le statut de " minorité " au Pays-Bas. Ces différences intériorisées par les populations concernées créent des identifications qui rendent encore plus difficile la définition d'intérêts communs et les stratégies d'une action commune.

Par ailleurs, les relations historiques privilégiées entre les anciennes colonies et les pays d'installation posent une autre difficulté d'identification avec les autres populations immigrées. Les associations algériennes, par exemple, semblent beaucoup moins motivées que les autres à élaborer des réseaux en dehors de la France, sauf avec le pays d'origine( ${ }^{12}$ ). Bien entendu, les liens familiaux des migrants avec ceux restés au pays persistent ainsi que les liens culturels, sociaux et même politiques avec les pays de l'autre rive de la Méditerranée. Mais pour eux, le transnational est seulement synonyme de trans-méditerranéen. 
Du coup, les efforts de coordination portent sur la recherche d'une revendication commune : la citoyenneté européenne. Du point de vue légal est citoyen européen toute personne ayant la nationalité d'un des pays de l'Union Européenne. Mais la représentativité recherchée par les migrants se situe au-delà de ce statut légal. Même si, en principe, la recherche d'une représentativité renvoie à la nationalité actuelle, elle s'exprime aussi à partir d'une situation d'immigration qui renvoie tantôt à la nationalité d'origine (pour ceux qui ont acquis la nationalité du pays de résidence), tantôt à une identité religieuse, notamment musulmane, qui n'est pas indépendante de l'identité nationale (actuelle ou d'origine).

\section{LA SUPRANATIONALITÉ ET LES RÉSEAUX FORMELS}

Les institutions supranationales aident les populations immigrées à élaborer des réseaux transnationaux en Europe. Tout comme les États des pays membres, les institutions européennes mobilisent leurs ressources pour créer une certaine compétition entre différentes associations locales, nationales, qu'elles soient isolées ou organisées en fédérations d'associations. Cette compétition portant sur leur représentativité s'appuie donc sur la nationalité actuelle ou d'origine, alors même que les activités des associations relèvent, en principe, du social. Par exemple, le Parlement Européen a réuni des associations d'immigrés sous une nouvelle structure appelée Forum des Migrants. D'après le chargé du suivi du Forum des Migrants à la Commission des Communautés Européennes, le but serait d'obtenir pour les ressortissants des pays tiers " les mêmes opportunités et les mêmes droits que les autochtones et redresser ainsi le manque démocratique "('3). Font partie du Forum des Migrants, en principe, les associations d'immigrés $\left({ }^{14}\right)$. La nationalité qu'elles " représentent " est donc le critère principal de sélection. Leur capacité d’organisation (multiplicité des nationalités représentées, nombre de filiales, étendue des réseaux), la pluralité des secteurs qu'elles recouvrent et leur représentativité dans le pays d'immigration importent autant que la nationalité qu'elles représentent. C'est ainsi que les associations de jeunes issus de l'immigration maghrébine en France ou celles de Black en Grande-Bretagne figurent aussi dans le Forum des Migrants.

Les critères de choix ne sont pas sans ambiguïté. Cette ambiguïté provient de la définition même d'une identité européenne. En effet, les critères de sélection des associations qui feraient partie du Forum font apparaitre plusieurs contradictions, notamment dans la définition des concepts d'immigré, d'étranger, de minorité. Ainsi, le terme d'immigré, qui renvoie surtout à des représentations à partir d'une condition sociale réelle ou imaginaire( ${ }^{15}$ ), est utilisé par le Forum des Migrants dans le sens de populations immigrées d'origine non-communautaire, donc opposé au "national-européen ". Mais en réalité, s'agit-il uniquement d'une population qui n’a aucun droit politique ni dans le pays de résidence ni en Europe du fait de sa nationalité (extra-communautaire) ? S'agit-il d'une population issue de l'immigration, qui, compte tenu des différences dans les lois sur la citoyenneté dans les pays de la Communauté, a acquis la nationalité du pays d'établissement (par le jeu du droit du sol, en France par exemple) ou des populations qui, compte tenu des relations coloniales, ont la nationalité du pays d'établissement, mais subissent les mêmes rejets que les étrangers de droit à cause d'une identité religieuse, ethnique ou raciale, (une partie de la population d'origine maghrébine en France, les 
"Blacks " en Grande-Bretagne, les Surinamiens, les Indonésiens aux Pays-Bas)? Pour les Blacks en Grande-Bretagne par exemple, qui ne se reconnaissent pas dans le statut d'immigré, la "couleur" est autant facteur d'exclusion que le noncitoyenneté. Ou tout simplement s'agit-il de toute population exclue de l'identité européenne par sa nationalité (actuelle ou passée), ou sa couleur, ou sa religion? Leur inclusion dans le Forum ne mettrait-il pas en évidence pour ces groupes leur exclusion de l'identité européenne en construction ? Dans tous les cas, leur présence dans le Forum leur confère un statut de minorité créé et fabriqué par le Parlement Européen.

Ainsi, le Parlement Européen reproduit les "modèles nationaux " dans la représentation des migrants en Europe. La supranationalité n'est en réalité que la projection des idéologies nationales et pose de ce fait le problème d'identification des militants associatifs dans les différents pays européens.

\section{IDENTIFICATION ET RÉSEAUX INFORMELS}

Lidentification des migrants n'est pas assurée par les initiatives du Parlement Européen, surtout si la volonté de représentativité repose sur une identité construite pour s'intégrer dans l'espace public européen. Dans une Europe qui se présente comme une arène où concourent les réseaux, des réseaux informels se développent en parallèle aux réseaux formels. C'est l'identité présumée d'origine, mais plutôt construite en situation d'immigration, qui constitue le nœud du réseau informel. L'islam en est le noyau dur. La religion institutionnalisée par les associations forme les maillons de la chaîne transfrontalière. Les représentants des associations islamiques opèrent en liaison avec les pays d'origine ou avec l'aide des organismes internationaux émanant des pays du Golfe, ou les deux à la fois. Ces réseaux s'insèrent dans le système européen et, tout comme au niveau local dans différents pays, font concurrence aux associations dites socio-culturelles et laïques. Les pays d'origine essaient de mobiliser les migrants pour obtenir une reconnaissance de leur pays (extra-communautaire) auprès des instances européennes. Par l'intermédiaire des circuits consulaires, ils soutiennent les initiatives des migrants dans les actions locales pour l'enseignement de la langue d'origine ou même pour l'ouverture de lieux de culte ou d'، écoles communautaires». En renforçant les particularismes nationaux, les États d'origine réactivent les allégeances de leurs ressortissants et contribuent à la création d'une communauté transnationale. Quant aux organismes internationaux qui cherchent à promouvoir l'islam, ils mobilisent des fonds pour dépasser la diversité nationale des musulmans installés en Europe, diffuser une identification unique dans la religion et créer ainsi une solidarité transnationale fondée sur l'islam.

Lidentification à l'islam pour une action commune au niveau européen se heurte à une autre difficulté qui provient de la diversité des nationalités, des sectes, des groupes ethno-culturels qui constituent la population musulmane en Europe. Cette diversité se reflète dans la multiplicité des associations locales, nationales, et même dans la présence de plusieurs fédérations d'associations religieuses dans les différents pays d'installation. De fait, chacune des organisations réunit le plus souvent une groupe national (turc, indien, marocain, algérien) ou une confrérie (soufisme). Dans un souci de représentation au niveau européen, certaines se présen- 
tent comme " multi-nationales ", car elles essaient de rassembler plusieurs nationalités, mais aussi transnationales, car représentées dans différents pays. Par ailleurs, certaines affichent une identité pacifique et se comportent comme des missionnaires pour encourager l'adhésion et la foi des Musulmans : c'est le cas par exemple de l'association Foi et Pratique (16). D'autres en revanche énoncent ouvertement la force politique de l'islam dans le système international. Mais dans la plupart des cas, les associations restent cantonnées à la nationalité qu'elles représentent, plus encore, aux partis politiques dont elles sont les porte-paroles en Europe. Un des exemples est l'Organisation Européenne de la Vision Nationale (AMGT), mouvement européen du parti religieux turc ; elle dispose à l'heure actuelle de 28 bureaux dispersés en Europe (dont dix en Allemagne). L'organisation développe sa " vision multinationale " en accueillant, notamment en Allemagne, des demandeurs d'asile algériens et bosniaques, pour manifester sa solidarité avec le FIS dans le premier cas et sa solidarité avec un peuple musulman européen, dans le second.

L'ensemble du réseau transnational fondé sur l'islam se présente surtout comme une structure d'accueil au nom d'une identification religieuse ou politique, ou les deux à la fois. L'objectif est d'aboutir à la reconnaissance de l'islam en Europe, plutôt qu'à l'existence d'un Islam européen. Les actions dans ce sens s'intègrent dans la formation d'une " minorité " religieuse. D'après $R$. Leveau, la Cour de Strasbourg sera amenée à l'avenir à pronocer des arrêts de principe prenant appui sur la Convention des Droits de l'Homme, qui reconnaît la liberté d'exercice des droits religieux. Ces arrêts établiront sans doute une certaine unité de comportement, régulant le fonctionnement dans leur diversité des institutions et des Cours amenées à gérer dans la pratique la constitution d'un Islam européen( ${ }^{17}$ ).

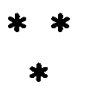

Les réseaux de solidarité des citoyens non-européens, réseaux fondés sur des identifications nationales et religieuses créent dans le cadre de la construction de l'Europe Unie une situation triangulaire : les États-nations les uns vis-à-vis des autres, chacun des États-nations face à la construction européenne et les immigrés (non-européens) à la fois face aux États-nations et au nouvel espace politique. L'ensemble des interactions renvoie au débat sur la définition de la citoyenneté et de l'identité européenne.

Les débats sur l'identité européenne relèvent surtout de la place accordée à des identités nationales, des identités régionales, des identités culturelles et religieuses. Chacune de ces identités se redéfinit par des jeux d'interaction et d'identification complexes qui conduisent surtout les philosophes à essayer de saisir l'histoire en cours et définir de nouveaux concepts qui dépassent les modèles nationaux $\left.{ }^{18}\right)$. Les interrogations portent surtout sur la reconnaissance des cultures nationales pour former une culture européenne commune. Une telle perspective définit l'Europe comme l'Europe des nations pour les États membres, l'Europe des États pour des populations en situation de " minorité " dans certains pays, et l'Europe des visas pour les étrangers installés dans différents pays.

Les militants se mobilisent face à une Europe fermée, une Europe des visas. Leurs actions pour élaborer des réseaux transnationaux apparaît comme une 
réponse aux replis identitaires des nations, exprimés plus ouvertement lors des débats sur le traité de Maastricht. Cela entraîne une perte de confiance en l'universalité des États-nations et les fait recourir à une autre conception de l'universalité qui serait un espace où les étrangers, résidents européens, feraient partie de la pluralité des cultures, au même titre que les identités nationales, qui la composent. Telle est la représentation de l'Europe. La recherche d'une représentativité au niveau européen par le biais des associations apparaît comme un moyen de faire face à des identités nationales ancrées dans la mémoire et l'histoire, de se constituer en " communauté transnationale " en réaction aux sentiments " communautaristes " des nations. Non seulement leur mobilisation pour développer des réseaux transnationaux leur confère le droit de participer à la construction politique de l'Europe, mais aussi les projets européens des États-nations leur procurent l'espoir de participer à la construction de l'Europe et de contribuer à forger son identité. Tout comme aux États-Unis, pays d'immigration dès sa constitution, les vagues successives prirent part à la définition de la nation américaine.

Ainsi l'Europe, ce nouvel espace politique à identité incertaine accélère l'urgence d'une redéfinition des concepts de citoyenneté et de nationalité qui adaptés à des États-nations ne correspondent plus à la nouvelle réalité. Même si la nationalité, telle qu'elle est définie par les critères du Forum des Migrants, se réfère à la nationalité d'origine, renforce les identifications nationales et religieuses extraeuropéennes et revêt un aspect ethnico-religieux, la citoyenneté, elle, renvoie au droit de la cité qui se définit comme un "droit " de participation, ce qui implique, dans le contexte européen, une identification culturelle avec la nouvelle " communauté de destin ". 


\section{Notes et références bibliographiques}

(1) Sources SOPFMI-OCDE, Eurostat, INED. Parmi cette population on compte 820000 Algériens, 516000 Marocains, 200000 Tunisiens en France, 1800000 Turcs en Allemagne, une importante population d'origine indienne $(689000)$, antillaise $(547000)$ et pakistanaise $(406000)$ en GrandeBretagne, 210000 Turcs et 165000 Marocains aux Pays-Bas. Bien entendu ces données statistiques ne tiennent pas compte des individus, qui malgré leur statut légal de citoyens des pays de résidence, sont toujours perçus comme culturellement étrangers, ou " minorités " (une partie des Maghrébins en France, les populations du Commonwealth en Grande-Bretagne, les Surinamiens, les Indonésiens aux Pays-Bas).

(2) J. Rex, Ethnic Identity and Mobilisation in Britain, CRER, Monographs in Ethnic Relations, no 5 .

(3) Par exemple, les migrants venus de Turquie négocient la reconnaissance de leur nationalité en se mobilisant pour la double nationalité en Allemagne et la reconnaissance de lislam en se joignant aux actions des autres populations musulmanes en France, la nationalité et la religion étant les éléments constitutifs du noyau dur de lidentité des Tures. Pour plus de détails of. R. Kastoryano, "Être Turc en France et en Allemagne " in CEMOTI, n" I3, 1992.

(4) C. Neveu montre que parmi les associations dans la région Roubaix-Tourcoing, les associations religieuses sont nombreuses, mais peu visibles, et les associations maghrébines sont faibles dans leur mobilisation et leur impact sur la population concernée. Les Maghrébins constituent $40 \%$ de la population immigrée avec une forte proportion d'Algériens, mais le nom des associations n'indique pas l'origine nationale de ces institutions.

C. Neveu "Éléments d'analyse à propos d'un exemple local : La région de Roubaix-Tourcoing ", in Rapport pour la Convention d'étude entre le Ministère de la Recherche et de l'Industrie et le Centre d'Êtudes et de Recherches Internationales sur Formes de Solidarités dans l'espace européen ; Paris, avril 1992.

(5) M.-A. Diop, "Une radio communautaire : Radio Gazelle de Marseille ", Rapport cité.

(6) M. Diop, R. Kastoryano, "Les associations islamiques en Ile de France " in Revue Européenne des Migrations Internationales, vol. 7, $\mathrm{n}^{\circ} 3$, 1991, pp. 91-119.

(7) Entretien avec le Délégué Régional du FAS à Metz en mai 1992.

(8) J. Rath, K. Groenendijk et R. Penninx, "The Recognition of Islam in Belgium, Great-Britain and Netherlands " in New Community, 18(1), pp. 101-114.

(9) R. Detalle, L'Islam à Berlin-Ouest, Mémoire présenté pour le DEA d'études politiques sous la direction de R. Leveau, Paris 1990, p. 79.

(10) J. Rex, op. cit.

(11) J. Habermas, Immigration et chauvinisme du bien-être, in Revue Nouvelle, no 10, 1992. Voir aussi, L.immigration et la citoyenneté dans une nouvelle Allemagne, in Courrier International. mars 1993.

(12) R. Leveau, le sabre et le turhan. Paris, éd. François Bourin, 1993.

(13) Objectif déclaré par le Forum des Migrants, cf. C. Neveu, "Citoyenneté ou racisme ", in Revue Européenne des Migrations Internationales, dans ce même numéro.

(14) I.e Conseil de l'Europe, avec l'appui d'organisations non gouvernementales, notamment les Églises (la Commission des Églises pour les Migrants en Europe, le World Council of Churches) et des institutions comme le Centre Européen du Travail (European Centre for Work) ou l'Euro Citizen Action Service, ainsi que le CIEMI (Centre d'Information et d'Etudes sur les Migrations Internationales) en France, a tenté d'établir une liste d’associations de migrants dans les douze pays de la Communauté pour constituer le Forum des Migrants ; J. King, "Ethnic minority communities and the politics of Europe ", in Revue Européenne des Migrations Internationales. Voir aussi, C. Neveu, op. cit. 
(15) Cf. A. Sayad, L'immigration ou les paradoxes de l'altérité, Bruxelles, Ed. Universitaires, 1991.

(16) G. Kepel, Les hanlieues de I'Islam, Paris, Seuil, 1987.

(17) R. I eveau, "I Islam en Europe ", in Formes de solidarité dans l'espace européen. Rapport de la convention d'études entre le MRI et le CERI, Paris, 1992.

(18) Parmi les penseurs les plus connus, Habermas propose un "patriotisme constitutionnel " qui doit " se relier à des principes juridiques universalistes uniques à partir des perspectives différentes imprégnées dans des histoires nationales ", J.-M. Ferry développe le concept de " postnational " qui souligne la différence entre nationalité et citoyenneté, " de sorte que la pluralité nationale soit compatible avec l'unité politique ", in J. Lenoble et N. Dewandre (dir.) L'Europe au soir du siècle : Identité et démocratie. Paris, Ed. Esprit, Seuil, 1992. 
Mobilisations des migrants en Europe : du national au transnational

\author{
Riva KASTORYANO
}

Il est devenu habituel d'opposer des "modèles" nationaux lorsqu'il s'agit de comparer les formes d'organisation et d'intégration des migrants des années 1960 installés dans différents pays européens. Ces modèles fondés sur les traditions politiques et les relations que chacun des états entretient avec les populations en situation de minorité (religieuse, ethnique, raciale) influencent la mobilisation des populations en question. En effet, leurs modes de mobilisation dénotent dans chacun des pays l'intériorisation des normes et des conduites politiques propres à chacun des pays et soulignent dès lors la "nationalisation" de leurs actions. Or. la construction de l'Europe remet en cause la validité et la pertinence des modèles. Le mode de participation politique des populations issues de limmigration trouve un appui dans ce nouvel espace politique qui favorise les solidarités transnationales en fonction de la nationalité (actuelle ou d'origine), la religion. ou la "couleur". Les actions qui les accompagnent contournent les politiques nationales pour aboutir à une représentativité au niveau européen.

\title{
Transnational Solidarities of Migrants in Europe
}

\section{Riva KASTORYANO}

A comparative study on the settlement of immigrants in different European countries emphasizes the " models" that each country develops as the way to deal with a minority group. These models hased on each country's the political iraditions and its conception of nationhood and citizenship obviously has an effect upon immigrants, leading to their mobilization through the creation of voluntary associations. The result might be calles the "nationalisation" of their political action. Projects for European Unity however, challenge the validity of these national models. In fact, migrants or minorities (the terminology varies from one country to another) are developping new networks hased on their nationality (actual or of origin), their religion or race in order to promote their political participation heyond national boundaries. Their aim is the search for a representativity on a European level.

\section{Transnationale Solidaritäten der Migranten in Europa}

\section{Riva KASTORYANO}

Es ist üblich geworden, von " nationalen Modellen" zu sprechen, wenn es um den Vergleich der Organisations - und Integrationsformen von Migranten geht, die sich seit den 60er Jahren in verschiedenen europäischen Ländern niedergelassen haben. Diese Modelle, die auf politischen Traditionen und dem Verhältnis heruhen. das der Staat zu Bevölkerungsgruppen in der Position von Minderheiten (seien sie es aufgrund der Religion, der Ethnie oder der Rasse) unterhält, heeinflussen die Mobilisierung dieser Gruppen. Ihre Mobilisierungsformen neisen in jedem Land auf die Interiorisierung der jen'eiligen politischen Normen und Verhaltensregeln hin und sind so ein Zeichen der "Nationalisierung " ihrer Aktionen. Die europäische Vereinigung stellt aher den Einfluss und die Prägnanz dieser Modelle in Frage. Die politischen Partizipationsformen der Migranten passen sich dem neuen politischen Feld an. das transnationale Solidaritäten bevorteilt, die sich aufgrund der Nationalität (der jetzigen oder der Herkunftsnationalität). der Religion oder der "Farhe " hilden. Die Aktivitäten. die damit einhergehen, umgehen die nationale Politik. um eine europäische Representativität zu erlangen. 